\title{
Evaluation of an image-guided navigation system in the training of functional endoscopic sinus surgeons. A prospective, randomised clinical study*
}

\author{
K. Stelter ${ }^{1}$, B. Ertl-Wagner ${ }^{2}$, M. Luz ${ }^{3}$, S. Müller ${ }^{4}$, G. Ledderose ${ }^{1}$, V. Siedek ${ }^{1}$, \\ A. Berghaus ${ }^{1}$, S. Arpe ${ }^{1}$, A. Leunig 1 \\ Department of Otorhinolaryngology at the Ludwig Maximilian University, Munich, Germany \\ 2 Institute for Clinical Radiology, University of Munich, Germany \\ 3 Specialist group for Industrial, Engineering and Organisational Psychology of the Technical University, \\ Berlin, Germany \\ 4 Innovation Centre Computer Assisted Surgery (ICCAS), Leipzig, Germany
}

SUMMARY

\begin{abstract}
Introduction: Many sinus surgeons report improved spatial orientation after using a navigation system. This study investigates the surgical, ergonomic and economic aspects of using a navigation system in training and teaching.

Materials and methods: Eight rhino-surgeons in training and 32 patients with bilateral diseases of the paranasal sinus system were included. After randomisation, one patient's side was operated on with a navigation system while the other side was operated on without navigation. It was monitored how often the surgeon used the navigation pointer and then changed the procedures. A standardised and validated interview recorded the cognitive load when using the navigation system and the application efficiency.

Results: The operations lasted on average 16 minutes longer with the navigation. Five paranasal sinuses could not be found in the control group without navigation. In only 10-13\% of cases did the surgical procedure change after the use of the pointer. Most of the surgeons admitted that particular steps of the operation were more reliable and safer to carry out with the navigation system. The general trust in the system rose in proportion to intraoperative accuracy and repeated use.

Conclusion: Overall, there was an overwhelming level of trust in the navigation system. Trainee sinus surgeons seeing their more experienced colleagues using a navigation device tend to overestimate the possibilities of the system and to underestimate the risks. The assistance system was used particularly effectively in the group of slightly more experienced surgeons. In this group, the additional expenditure of time was less and the navigation substantially contributed to reinforcing the anatomical sense of direction.
\end{abstract}

Key words: navigation, FESS, surgical training, perceived risk, workload

\section{INTRODUCTION}

The use of a navigation system in surgical training is currently controversial ${ }^{(1-3)}$. A survey by the University of Leipzig and Berlin found out that approximately $30 \%$ of all ear, nose and throat clinics in Germany have access to navigation systems, which are used in $90 \%$ of FESS ${ }^{(4)}$. Use of the systems in clinics varies considerably. Sometimes only highly complicated operations are navigation-supported, whereas in other cases the systems are used nearly daily, even for simple procedures.

The consequences of instrument navigation with experienced surgeons is assessed in overwhelmingly positive terms in the literature ${ }^{(4)}$. Most ear, nose and throat surgeons report improved spatial orientation, $45 \%$ better situation awareness and over $2 / 3$ assume better surgical results after using a navigation system on FESS ${ }^{(4)}$. At the same time, it is feared that using a navigation device in surgical training could be rather dangerous and lead to a loss of important anatomical knowledge and surgical expertise. Surgeons that use the device agree that instrument navigation should be used as often as possible and hence also with simple operations. Only in this way can 
familiarity with the system be achieved. In established centres, a navigation system enables up to $10 \%$ of OR time to be saved ${ }^{(4,7)}$. A complete evaluation of a navigation system should principally include technical, surgical, ergonomic and economic characteristics ${ }^{(1)}$ : the technical characteristics (accuracy and precision) of several navigation systems have been adequately studied by our working group ${ }^{(8-10)}$.

But surgical, ergonomic and economic features have until now not been researched in routine operations in surgical training. Specifically, the following questions should be answered:

\section{Surgical characteristics:}

- Does the trainee surgeon improve the postoperative outcome with the navigation (has he drained all the diseased paranasal sinuses)?

- Did the trainee surgeon change surgical procedures in routine operations because of the navigation (change of strategy)?

- Does the supervisor have to intervene in the operation process less frequently?

- Does the surgeon take more risks (risk homeostasis)?

- Does the trainee over-rely on the device or can the navigation lead to misdirection (excessive trust)?

- Did the surgeon perhaps lose skills because he allowed the system to do the work (surgical skill loss)?

Ergonomic characteristics:

- What are the system's controllability, expectation conformity and self-description capabilities?

- Does it affect the situation awareness?

- Is there a reasonable ratio between the cost of the device and its utility (effort to engage)?

- Can the tasks of the surgeon be appropriately carried out with the system (skill set requirements)?

- Does the system reduce cognitive and physical demands and how does the surgeon handle the increasing workload with the pictorial information offered (workload shift)?

Economic characteristics:

- How high is the additional expenditure of OR time in using the device in standard situations?

- What is the cost of using the navigation device in training?

\section{MATERIALS AND METHODS}

The randomised, single blinded clinical study was planned and carried out in accordance with the GCP guidelines and CONSORT (Consolidated Standards of Reporting Trials) Statement ${ }^{(11)}$ of 2008.

Thirty-two patients with bilateral diseases of the paranasal sinus system (chronic sinusitis with or without polyposis nasi) were included between June 2009 and May 2010. In all operations, a navigation system was primarily not required, i.e. all landmarks could be detected without navigation by an experienced surgeon. Complicated anatomy and revision cases were excluded. The operations were carried out with 8 differ- ent operating surgeons in training under the supervision of 2 experienced rhinosurgeons. After a block randomisation, one patient's side was operated with the help of a navigation system (VectorVision compact ${ }^{\circledR}$ with laser registration, BrainLAB, Feldkirchen, Germany), the other patient's side was operated without navigation (intra-individual design). At the end of the operation, an evaluation was made with the navigation device and the supervising senior physician as to whether the operating surgeon had correctly operated on all the defined regions of the paranasal sinuses (Figure 1).

During the surgery, it was monitored how often the surgeon used the navigation pointer, on which part of the anatomy and how often he changed his surgical strategy. After the first and fourth (last) FESS, each surgeon filled out a standardised and validated questionnaire: the 'Human Factors Evaluation Questionnaire for Computer Assisted Surgery Systems (HFEQ-CASS).'

Intraoperatively, the following objective data were ascertained:

- Operation duration (beginning and end) of both sides separated from each other

- Required time for data transfer and referencing (surface matching by laser)

- Use of the pointer on different anatomical sides (Lamina Papyracea, skull base, sphenoidal sinus and the frontal recess)

- Change of surgical strategy based on information from the navigation device

- Postoperative drained paranasal sinuses right and left (outcome)

The HFEQ-CASS contains 38 items in two categories. The first question block (12 questions) consisted of

- Mental demands and work load (5 questions)

- $\quad$ Surgical results of the operation (1 question)

- Situation awareness (3 questions)

- $\quad$ Speed (1 question)

- $\quad$ Readiness to take risks (2 questions)

The first five questions were obtained from the Nasa Task Load Index ${ }^{(12)}$. The concept and questions on situation awareness were developed by Endsley and Kaber ${ }^{(13)}$.

The second question block covered surgical and ergonomic characteristics with 26 questions:

- Cross-checks before use of relevant structures (3 questions)

- Discovering malfunctions (1 question)

- $\quad$ Reduction of surgical sense of direction $=$ skill loss (2 questions)

- Application error = automation bias (1 question)

- Expectation conformity = usability (12 questions)

- Cost-benefit ratio (effort to engage) (1 question)

- Reliability (2 questions)

- $\quad$ Patient safety (2 questions)

- $\quad$ Trust (2 questions) 


\section{Statistics}

In the planning and analysis of this study a statistician of the institute of biometry, epidemiology and information processing was involved. Every participating surgeon had to do exactly four operations to cover all possibilities with the navigation system and sides:

- $\quad$ begin with the right side with the navigation system

- $\quad$ begin with the left side with the navigation system

- begin with the right side without the navigation system

- begin with the left side without the navigation system.

To test if the use of the navigation system had significant impact on the surgeons' perceived workload and performance as compared to unsupported surgery, ratings on the scales of the first part of the questionnaire were tested for significant deviations from the midpoint of the scale, 3 , by the one-sample $t$-tests. A significant deviation from the neutral midpoint meant a benefit with $>3$ or a disadvantage with $<3$ in use of the navigation system compared to the other patient's side without the navigation system.

For the second part of the HFEQ-CASS (questions 13-38), the same test (double-sided t-test compared with 3) was basically used. We decided to apply the Bonferroni ${ }^{(14)}$ correction in the present study only to tests including items or scales focusing on aspects of the same human factors. This corresponds to a familywise approach to statistical testing in other settings. For example, changes of workload were analysed by six dependent $t$-tests (one test each for the overall workload score and the five single dimensions of workload considered). In this case, an effect of a single $t$-test was only considered to be significant if $\alpha$ $\leq 0.05 / 6=0.008$. In accordance with this procedure, effects will only be reported as significant of the given $\alpha$-level if their probabilities are equal to or smaller than the Bonferroni adjusted $\alpha$. The results were evaluated with SPSS 14 (Chicago, IL, USA). The graphic treatment was done with SigmaPlot 2000. A prospective power analysis was not carried out since there was no data from preliminary investigations, which could relate to possible differences and mean variations.

\section{RESULTS}

\section{Patient collective}

In accordance with the study design, all 32 patients were operated on by 8 operating surgeons. After information was provided on participation in the study with an explanation of the functioning of the navigation device, 18 (56\%) patients opted to be operated on with a navigation device, $14(44 \%)$ patients said it did not matter whether they were operated with or without a navigation device. The average age of the patients was 46 years ( $\mathrm{SD}=16$ years). All 32 patients suffered from chronic sinusitis on both sides, 17 patients had nasal polyps bilaterally, 16 patients also had septal deviation, 5 patients suffered from Samter's Triad.

In total there were 157 diseased paranasal sinuses (right and left): 60 anterior ethmoid cells and maxillary sinuses (= $92 \%$ of the patients), 54 posterior ethmoid cells ( $=52 \%$ of the patients), 25 frontal sinuses ( $=30 \%$ of the patients), 18 sphenoidal sinuses ( $=22 \%$ of the patients).

\section{Drop outs}

In this study, all 8 subjects (surgeons) completed the prescribed number of operations. There were no dropouts among the patients after inclusion and signed patient information. Accuracy and precision were measured intraoperatively by target registration error (TRE) before each application of the navigation system and after patient registration. Only if the TRE was acceptable, the referencing was accepted. In two cases, registration was impossible, because the data record was too roughly layered. In two other cases, the data record could not be transmitted to the navigation system, because the CT data in DICOM format was carried by an external radiologist to the CD-ROM without the file ending $\mathrm{dcm}$.

\section{Postoperative Outcome}

The 157 diseased paranasal sinuses were equally distributed on both sides. During the operation, 80 paranasal sinuses were operated with the help of the navigation and 77 without. The supervisor had to intervene during the operation as often in the study group as in the control group.

At the end of surgery, the supervisor found with the aid of the navigation a total of 5 paranasal sinuses, which had not been opened without the navigation, and 2 sphenoidal sinuses, which had not been opened even with the use of the navigation (Table 1). The supervisor opened the infected sinuses by himself to complete the operation.

\section{Frequency of pointer use and change of strategy}

The pointer usage varied a lot between individuals. Not only the operating surgeon, but also the actual situation and the patient played a decisive role.

The change of surgical strategy is the most important aspect in the use of surgical assistance systems. In only $10-13 \%$ of cases, the surgical procedure was changed. (Table 2). In these cases, it could not be differentiated whether the operating surgeon used the navigation to verify or correct his previously assumed instrument position.

At the end of each operation, the surgeon had to state on a visual analogue scale (VAS) whether he operated with the navigation on structures, which he would not have operated on without the navigation. With a mean value of $55 \mathrm{~mm}$ (SD = $34 \mathrm{~mm}$ ), most surgeons believed that they would have done the same steps of the operation with the navigation as without the navigation.

\section{Expanding the indication spectrum}

The HFEQ_CASS had three questions indirectly relating the readiness to expand the indication spectrum by using the navigation. The answers showed highly significantly ( $p=0.00000093$ ) that the trainee surgeons thought that particular steps of the operations were taken more reliably and safely with the navigation system and therefore they were more likely to try them. 


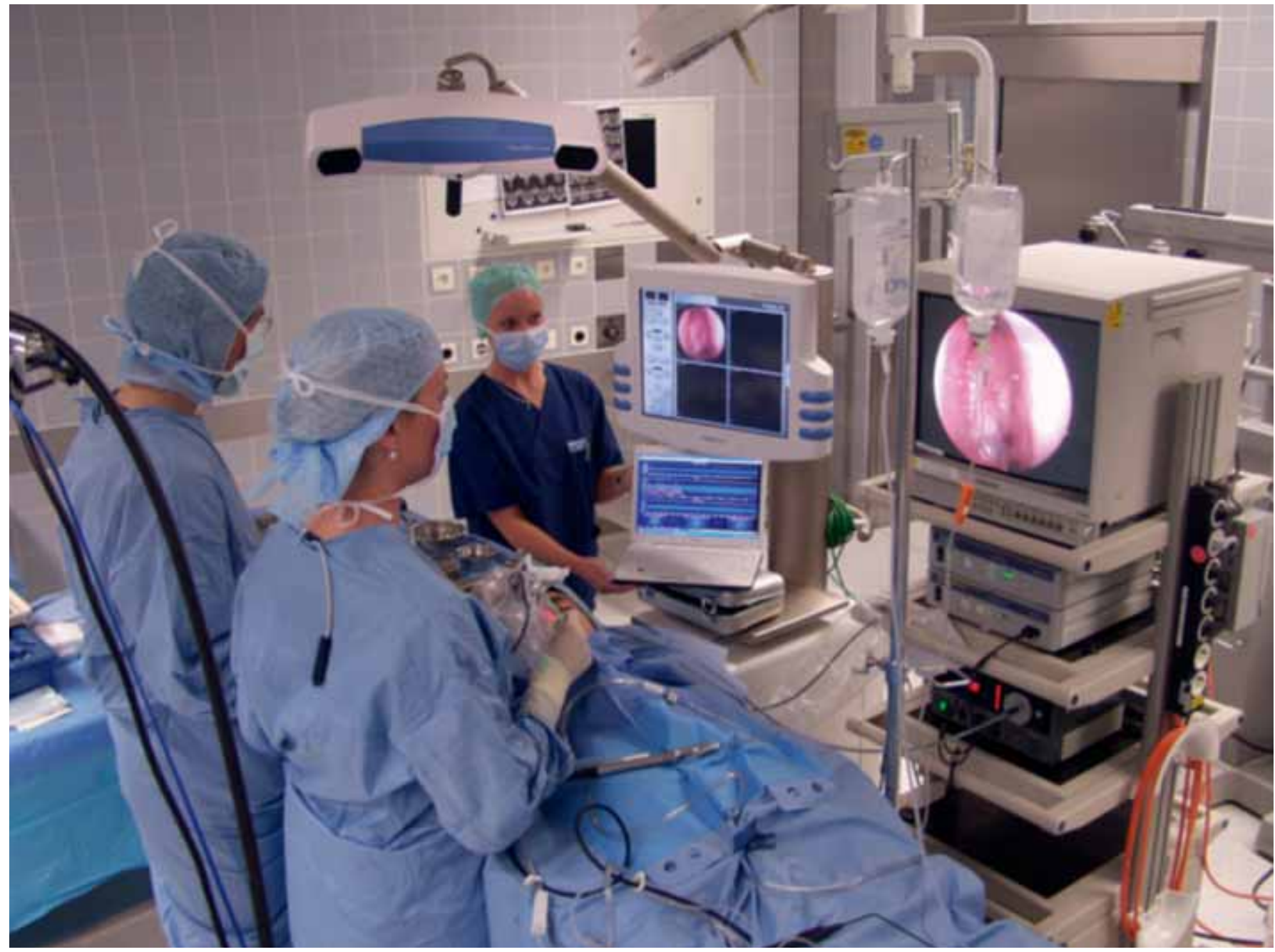

Figure 1. Setting in the OR. The operating surgeon carries out the FESS guided by the 19 " monitor. To the left side is the VectorVision compact ${ }^{\mathbb{B}}$ placed. All objective data was recorded by a third person 'on the fly' with the study notebook (centre).

\section{Risk management and complications}

Intraoperative complications included bleeding, which obscured the surgical view $(n=4)$ and injury to the lamina papyracea and periorbita $(\mathrm{n}=2)$. All complications were distributed equally in the study group (with navigation) and the control group (without navigation). There were no major complications (CSF-leaks, heavy arterial bleeding, injury to the N. opticus or orbital cavity muscles). However, the questionnaire revealed a significantly increased willingness to take risks when using the navigation system.

\section{Over-reliance and over-trust}

Both, after the first operation with the navigation system and after the fourth operation with the system, all surgeons thought that paranasal sinus operations could be more safely carried out, and all surgeons trusted the system. On the other hand, many surgeons didn't share the opinion that the system provides greater protection from faulty treatment.

The important issue of trust in the system was additionally raised after each operation based on a VAS. In this way, the operating surgeon had to indicate whether he trusted the navigation system or not. Depending on the intraoperative accuracy of the system this produced extremely varied values. Overall, in 32 operations all operating surgeons trusted the navigation system in over $90 \%$ of cases (Figure 2).

The high trust and feeling of safety must be compared with the probability of an operating error due to the navigation system. The HQEF-CASS asked how often the surgeon checked accuracy (cross check) and whether he/she blindly trusted the device's information. The beginner surgeons believed that they would quickly notice a malfunction of the system in critical situations. At the same time, many admitted that the system was not regularly checked for accuracy especially after the 4th operation. Even when the trust in the system was very high, most surgeons indicated that they trusted their own impressions rather than the system's information.

\section{Surgical skill loss}

No trainee surgeon thought of a loss of surgical skills when using the navigation system. On the contrary, almost all surgeons reported a better anatomical understanding with the additional information of the image-guided navigation. 


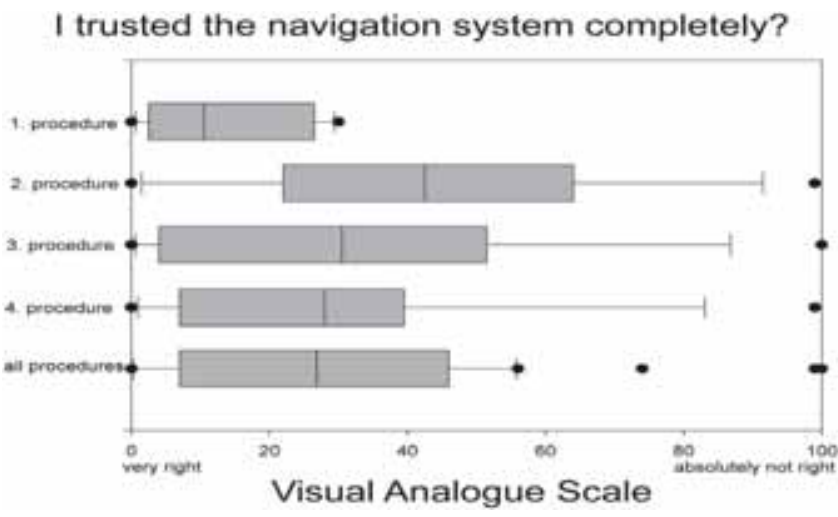

Figure 2. Box plots filled out by beginner surgeons with median, $65 \%$ and $95 \%$ percentile and exceptions to total trust in the system. $0 \mathrm{~mm}=$ maximum trust, $100 \mathrm{~mm}=$ no trust .

However, three experienced operating surgeons admitted that beginner surgeons would possibly develop less of a surgical sense of direction if they worked with the navigation from the beginning.

\section{Ergonomic characteristics \\ Workload shift}

No significant difference between the control and study group was indicated thereby. This means that the surgeons felt no higher workload when using the navigation system.

\section{Usability}

Usability was perceived as positive. Results after the first operation were an average of 2.17 (SD: 0.77) [Scale: $1=$ very good usability, $5=$ very poor usability] and after the fourth operation even better with an average of 1.95 (SD: 0.82). The values indicated highly significantly a positive usability

\section{Situation awareness}

Situation awareness was recorded by 3 questions and was significantly better when using the navigation. The answer patterns after the first and fourth operations did not significantly differ from each other $(\mathrm{p}=0.39)$.

\section{Effort to engage}

All surgeons thought that the operational cost of the device was in proportion to the use; after the fourth operation even more than after the first operation.

\section{Economic characteristics}

The extent of additional operating room time with the use of a navigation device in standard situations is shown in Figure 3. Although the duration of the study side was 16 minutes longer on average, there were no significant differences either interindividually (patient to patient) or intra-individually (right
Duration of FESS in the study and control side

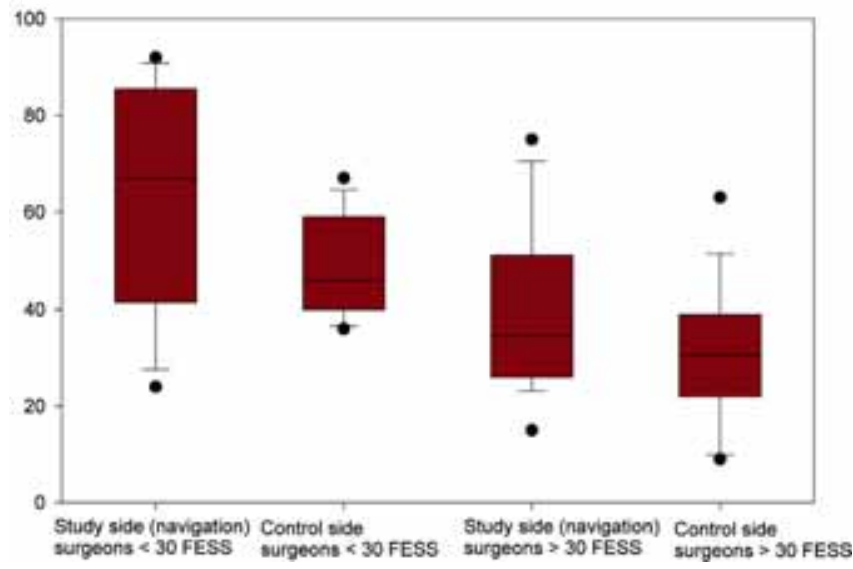

Figure 3. Box plots of the navigated side compared to the control side distributed to less experienced operating surgeons $(<30$ FESS operations; $n=4)$ and more experienced FESS surgeons $(>30$ FESS operations, $\mathrm{n}=4$ ).

against left patient's side) because of the wide variations in individual OR times. Particularly among the beginners $(<30$ FESS operations), there was a trend $(p=0.06)$ to extend OR time with the navigation (Figure 3 ).

\section{Cost of a navigation device}

The following prices are the individual list prices of the navigation device with the accessory used from BrainLAB in February 2010 in Euro:

Vector VisionII

$€ 74.000$

Softouch and Z-touch registration packet

$€ 29.400$,-

I-Plan ear, nose and throat planning software

$€ 15.500,-$

Pointer, headband, instrument adapter,

ICM, Steribox

$€ 18.830$,-

20 reference headbands with markers

$€ 550$,-

On-site installation and maintenance contract

$€ 8.450$,-

Total: $€ 146.730$,-

Added to this is the extended OR time when using the system for training from 16 minutes per group plus 17 minutes registration and boot up.

\section{DISCUSSION}

Since it is unclear what psychic demand actually is and this phenomenon also cannot be quantified with scientific methods, it seems reasonable to simply ask people how they subjectively evaluate the demand in a given work situation with informational content. The HFEQ-CASS was designed by the technical group for Industrial, Engineering and Organisational Psychology at the TU Berlin and the Innovation Centre Computer Assisted Surgery (ICCAS) Leipzig especially for evaluating cognitive load in using navigation systems in ORs. A check of internal consistency (Cronbach's $\alpha$ ) was possible based on the redundant method of enquiry and produced conclusions on the reliability of the questionnaire. 
In preliminary investigations of 213 surgeons, Cronbach's $\alpha$ produced a number between 0.69 and 0.83 (whereby $>0.7$ values are considered very reliable) ${ }^{(4)}$.

Filling out the HFEQ-CASS after each operation was not feasible in view of the scope. Hence, after each operation, the operating surgeons were only asked three specific questions, which were filled out based on a visual analogue scale (VAS). The three questions covered change in surgical strategy, perceived risk and trust in the system.

\section{Change in surgical strategy}

Relatively the navigation was used most often for finding the frontal sinus. Frequent use on the frontal sinus corresponds to expectations, since there are the greatest uncertainties and anatomic variations and even experienced paranasal sinus surgeons described finding the frontal sinus as the most demanding part of a FESS ${ }^{(17)}$.

A change in surgical strategy in only $10 \%$ of the cases is substantially lower than indicated in the literature ${ }^{(1)}$. The surgeons were prompted by the supervisor to use the pointer regularly to practice handling the navigation device. Therefore, the navigation was used very often to verify already known landmarks and only rarely contradicted the a priori information of the operating surgeon. In 10\% - 13\% of cases, however, the pointer position did not correspond to expectations and led to a change in surgical procedure. It was most often suspected that the frontal sinus was opened while, after checking, the naviga- tion showed that the operating surgeon was only looking into an agger nasi cell. A change in surgical strategy on the skull base was mostly indicated after the skull base was confused with cells from the posterior ethmoid bone, just as posterior ethmoidal cells were often confused with the sphenoid sinus. Whether the operating surgeon would have trusted the information from the navigation system to the extent of independently changing his surgical strategy even without the feedback from the supervisor remains a matter for speculation. Very interesting are the two sphenoid sinuses that were not opened/ found despite using the navigation (see Table 1). In both cases, the operating surgeons did not trust the navigation and did not open the sphenoid sinus so as not to harm the patient in any way. In both cases, the sphenoid sinus was then opened by the supervisor. On the other hand, in one case navigation was so helpful that the operating surgeon opened the frontal recess wide although this was not assessed as necessary by the supervisor. In doing this, the training surgeon was overestimating his expertise and the possibilities of the navigation and after the operation he recognised that his trust in the system may have been exaggerated.

\section{Trust in the system}

General trust in the navigation system was very high among most subjects from the beginning. After the first operation, all surgeons were ready to operate with the navigation system themselves. However, it was already clear to three surgeons

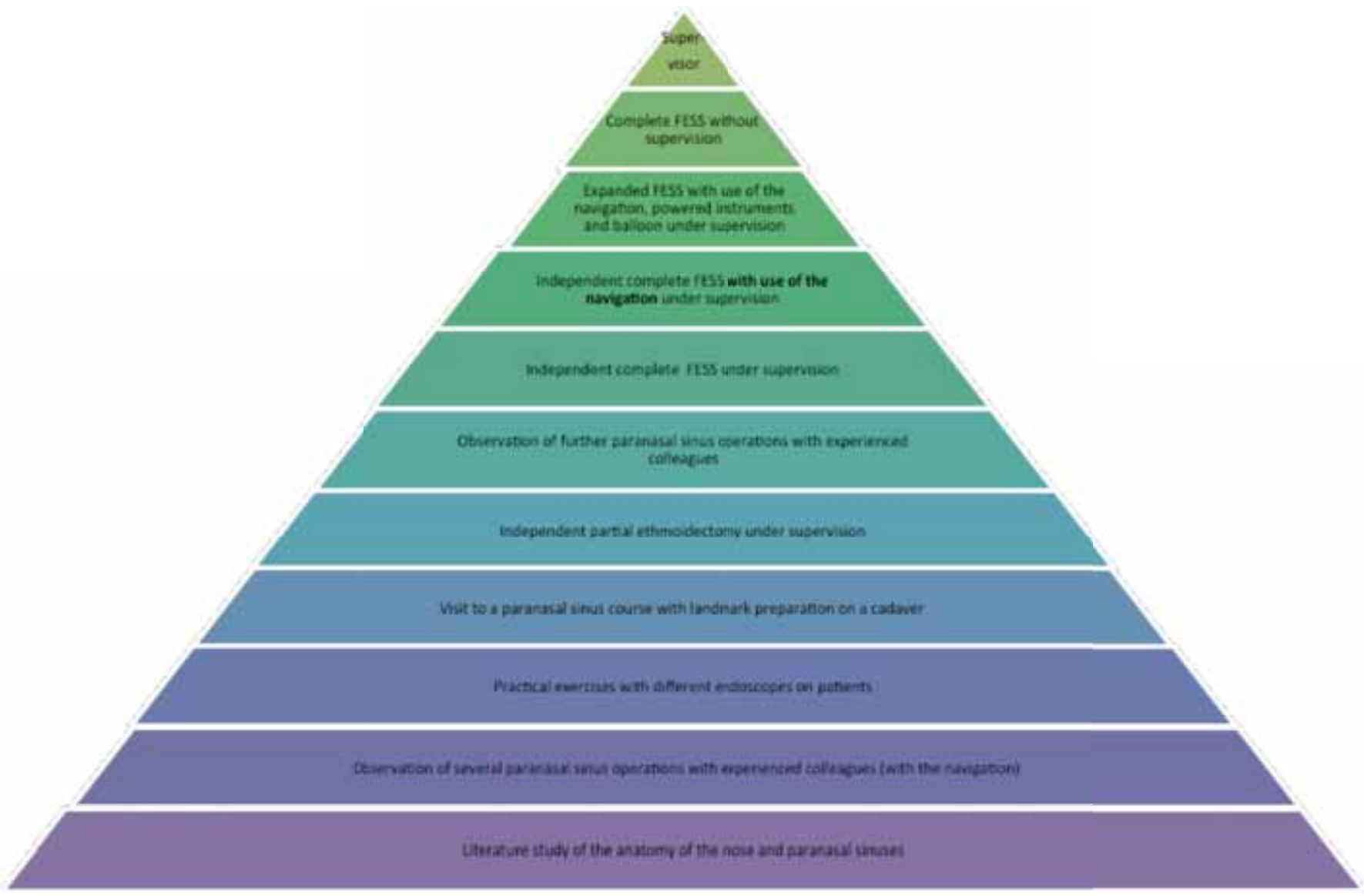

Figure 4. The Munich model of structured FESS training. 
after the fourth operation that a navigation system offers no protection against treatment failure but instead could lead to over-trust and wrong paths.

There was a direct relation between clinical accuracy and trust in the system. In this connection, it is interesting that, when specifically asked if they completely trusted the system during the operation, many surgeons did not trust the system because the deviation was too high. At some distance from the operation and in another context (by the HFEQ_CASS), all surgeons suddenly thought that operations could be done more safely and that there was greater protection from treatment failure. A special problem is the statement of four surgeons after the fourth operation that they no longer did regular cross checks. This is dangerous regarding the increased readiness to take risks with the perceived security provided by the navigation.

Experienced FESS surgeons only trust the system with increased frequency of use ${ }^{4}$. Whereas beginner surgeons who had already seen a navigation device used by experienced colleagues tended to overestimate the possibilities of the system and to underestimate the risks.

\section{Cognitive and physical workload}

Most operating surgeons indicated that they did not feel any additional workload from the navigation. This was partly because no surgeon (although anonymised) likes to admit that he/she is negatively affected by high technology in the OR. Another important aspect is that the navigation system was used without any time pressure and after systematic instruction under supervision in the context of this clinical study. Hence, most surgeons even found the navigation to be a mental relief and handling the technology as motivating instead of frustrating.

\section{Surgical skill loss}

In this study, the surgeons were forced to carry out a particularly precise operation plan on behalf of the CT findings preoperatively. These skills could be lost by using the navigation since it is possible to 'simply' operate and consult the navigation in critical situations without a detailed preoperative plan. However, no surgeon saw this as a risk to his own knowledge and skills, but some surgeons admitted that this could happen to younger colleagues in particular and estimated that use of the navigation could be critical for these colleagues.

\section{Time effort and costs}

Beginners need more time for operations than experienced surgeons, especially when using additional technology like the navigation. The period per group particularly depends on the level of experience of the surgeon and the difficulty of the anatomy. Hence, wide variations from 9 minutes to 90 minutes per group and side were not surprising. The extended operating time in using the system compared to the opposing group was on average 16 minutes plus 17 minutes registration and boot up. Assuming $€ 10,-/ m i n$ OR time ${ }^{(1)}$ additional training costs came to $€ 390$,- per use of the navigation system.
However, some steps (system boot up, data transfer and CT check up) are carried out by the scrub nurse or the surgeon before the start of the operation, which then does not extend incision to closure time. A considerable extension of OR time should be expected, especially when the navigation is used for the first time. By the second application, a lot of time was already being saved. In addition, during the first four applications pointer use was more rare, but also more effective. If this trend is to be continued, time saved when using the navigation should be expected ${ }^{(18)}$. This study unambiguously shows that effectiveness and time saved by regular use of the assistance system can be increased.

\section{Next steps}

An interesting question would be how often a supposedly simple paranasal sinus operation became so complicated that the operating surgeon would be thankful to have a navigation device on board. Most surgeons with access to a navigation device either use the technology very often and for many indications, or use it very rarely and only in extreme cases. To increase or reduce the frequency of use of such assistance systems to a reasonable level, further investigations are essential.

\section{CONCLUSION}

The navigation should have an assured place in training and teaching for paranasal sinus operations. Even if this new technology means extra costs, it was welcomed by all study participants (surgeons and patients). Good surgical training is initially expensive, but having a well-trained surgeon in the house is invaluable. The assistance system was used particularly effectively in the group of somewhat experienced operating surgeons (> 30 paranasal sinus operations). Additional expenditure of time was less and the navigation contributed substantially to enhancing anatomical sense of direction. Handling the device must be practiced in familiar environments, without pressure and under supervision. So it makes no sense to reserve this technology only for particular paranasal sinus surgeons for particularly difficult situations. In emergencies, the system can only be used effectively if it had previously been often used. Depending on their attitude to technology and level of training, operating surgeons are prejudiced in favour of or against this technology. These prejudices can be broken down by targeted use of the device in standard situations.

The Munich model of structured FESS training has scheduled regular use of the navigation since the results of this study. This is based on the training sequence in Figure 4.

The Munich training curriculum places particular emphasis on structured landmark preparation. The navigation can be helpful, particularly in finding the corresponding landmarks. Using this technology is, however, pointless without anatomical knowledge and an accurate idea of the course of the operation. The human supervisor can also not be replaced by a navigation device. As long as the above points are adhered to, it should not be feared that surgical skills will be lost because of using the navigation. 
Table 1. Missed paranasal sinuses with and without navigation. Additionally opened paranasal sinuses were paranasal sinuses, which should not have been opened according to the preoperative CT scan but which were opened anyway.

\begin{tabular}{lcr}
\hline & With navigation & Without navigation \\
\hline Missed paranasal sinuses & 2 sphenoid sinuses & 1 frontal sinus \\
& 3 sphenoid sinuses & 1 posterior ethmoid sinus \\
Additionally opened sinuses & 1 frontal sinus & 1 posterior ethmoid sinus \\
\hline
\end{tabular}

Table 2. Absolute values of the paranasal sinuses opened with the navigation in relation to pointer usage and change of surgical strategy.

\begin{tabular}{lcccc}
\hline & $\begin{array}{c}\text { Lam. papyracea }+ \\
\text { maxillary sinus }\end{array}$ & Posterior ethmoidal cells & Frontal sinus & Sphenoidal sinus \\
\hline Operated sinus & 60 & 54 & 25 & 18 \\
With navigation & 30 & 27 & 46 & 25 \\
Pointer-usage & 67 & 68 & 6 & 3 \\
Change of strategy & 7 & 9 & 0.13 & 0.12 \\
Ratio & 0.10 & 0.13 & & 25 \\
\hline
\end{tabular}

\section{ACKNOWLEDGMENTS}

BrainLab Sales, Feldkirchen, Germany supported the study by providing a second VectorVision compact ${ }^{\circledR}$ navigation device with complete Soft-Touch referencing set and all disposables free of charge for the duration of the study.

\section{CONFLICT OF INTEREST}

None.

\section{AUTHORSHIP CONTRIBUTION}

$\mathrm{KS}, \mathrm{AL}$ are the principal investigators of this study. They had the idea and wrote the paper. BEW prepared all the DICOM Files for navigation and contributed with her radiological point of view. ML, SM prepared and interpreted the HFEQ_ CASS. GL, VS included the patients, checked the informed consents and did all the postoperative nasal care. AB is the head of the department and was therefore responsible for the whole study. SA did her doctoral thesis with the results of the study. She collected and sorted all data (intraoperatively and the HFEQ_CASS) and did all statistical calculations.

\section{REFERENCES}

1. Strauss G, Koulechov K, Rottger S, et al. Evaluation of a navigation system for ENT with surgical efficiency criteria. Laryngoscope. 2006; 116: 564-572.

2. Mukherjee M, Siu KC, Suh IH, Klutman A, Oleynikov D, Stergiou N. A virtual reality training program for improvement of robotic surgical skills. Stud Health Technol Inform. 2009; 142: 210-214.

3. Strauss G, Bahrami N, Possneck A, et al. Evaluation of a training system for middle ear surgery with optoelectric detection. HNO. 2009; 57: 999-1009.
4. Manzey D, Rottger S, Bahner-Heyne JE, et al. Image-guided navigation: the surgeon's perspective on performance consequences and human factors issues. Int J Med Robot. 2009; 5: 297-308.

5. Strauss G. Computer-assisted surgery of the frontal skull base. HNO. 2009. 57: 990-997.

6. Strauss G, Limpert E, Strauss M, et al. Evaluation of a Daily used Navigation System for FESS. Laryngorhinootologie. 2009; 88: 776-781.

7. Neumuth T, Trantakis C, Riffaud L, Strauss G, Meixensberger J, Burgert O. Assessment of technical needs for surgical equipment by surgical process models. Minim Invasive Ther Allied Technol. 2009; 18: 341-349.

8. Stelter K, Andratschke M, Leunig A, Hagedorn H. Computerassisted surgery of the paranasal sinuses: technical and clinical experience with 368 patients, using the Vector Vision Compact system. J Laryngol Otol. 2006; 120: 1026-1032.

9. Stelter K, Ledderose GJ, Tschiesner U, Matthias C, Spiegl KE. Clinical Application of a New Dental Reference System for Computer Assisted Surgery at the Lateral Skull Base. Open Otorhinolaryngol J. 2008; 2, 49-56.

10. Siedek V, Stelter K, Betz CS, Berghaus A, Leunig A. Functional endoscopic sinus surgery - a retrospective analysis of 115 children and adolescents with chronic rhinosinusitis. Int J Pediatr Otorhinolaryngol. 2009; 73: 741-745.

11. Hopewell S, Clarke M, Moher D, et al. CONSORT for reporting randomised trials in journal and conference abstracts. Lancet. 2008; 26: 371: 281-283.

12. Hart SG, Staveland LE. Development of a multi-dimensionalworkload rating scale: Results of empirical and theoretical research. In: P.A. Hancock \& N. Meshkati, ed. Human mental workload. Amsterdam, The Netherlands: Elsevier; 1988: 139-183.

13. Endsley MR, Kaber DB. Level of automation effects on performance, situation awareness and workload in a dynamic control task. Ergonomics. 1999; 42: 462-492.

14. Abdi H. The Bonferonni and Sidak corrections for multiple comparisons. In: Salkind N., ed. Encyclopedia of Measurement and Statistics. Sage Publications: Thousand Oaks; 2007; 103-107. 
15. Bartenwerfer H. A few practical consequences from the activation theory. Z Exp Angew Psychol. 1969; 16: 195-222.

16. Rohekar G, Pope J. Test-retest reliability of patient global assessment and physician global assessment in rheumatoid arthritis. $\mathrm{J}$ Rheumatol. 2009; 36: 2178-2182.

17. Stammberger $\mathrm{H}$. The evolution of functional endoscopic sinus surgery. Ear Nose Throat J. 1994; 73: 451, 454-455.

18. Strauss G, Koulechov K, Rottger S, et al. Clinical efficiency and the influence of human factors on ear, nose, and throat navigation systems. HNO. 2006; 54: 947-957.
Klaus Stelter, MD

Dept. of Otorhinolaryngology, Head and Neck Surgery

Grosshadern Medical Centre of Ludwig-Maximilians-

University

Marchioninistr. 15

81377 Munich

Germany

Tel: +49-89-7095 0

Fax: +49-89-7095 6869

E-mail: Klaus.stelter@med.uni-muenchen.de

\section{ADVERTISEMENT}

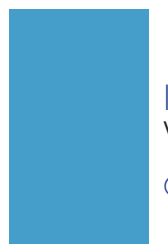

International Federation Facial Plastic Surgery Societies (IFFPSS)

VII International Congress

Congress President: Pietro Palma, MD

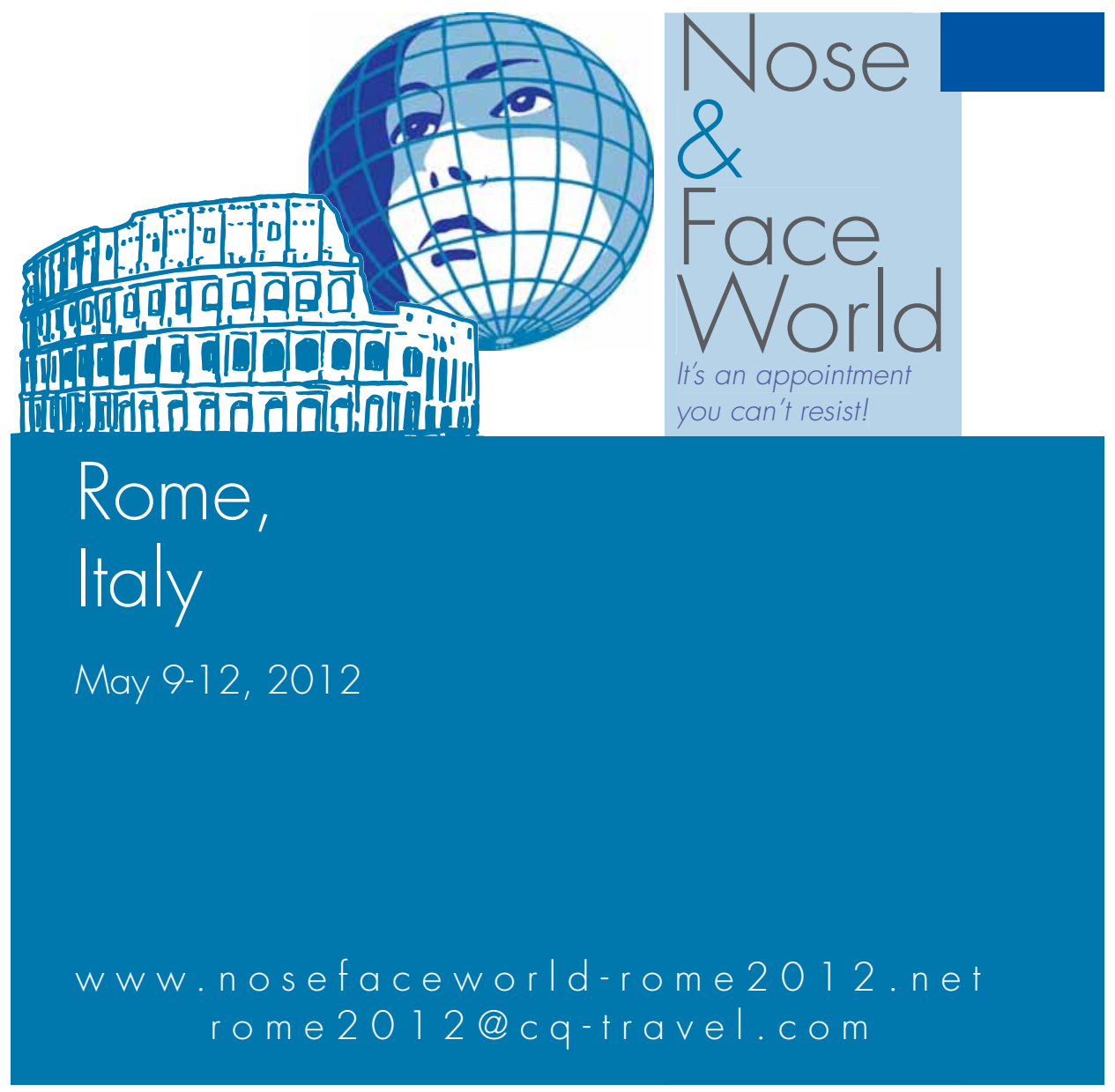

